\title{
Bivalirudin Versus Heparin During Percutaneous Coronary Intervention in Patients With Acute Myocardial Infarction
}

\author{
Hiten Patel ${ }^{\mathrm{a}, \mathrm{b}}$, Rana Garris ${ }^{\mathrm{b}, \mathrm{e}}$, Suchit Bhutanic, Priyank Shah ${ }^{\mathrm{d}}$, Upamanyu Rampal ${ }^{\mathrm{b}}$, \\ Rahul Vasudev ${ }^{\mathrm{b}}$, Gabriel Melki ${ }^{\mathrm{b}}$, Bader Abu Ghalyoun ${ }^{\mathrm{b}}$, Hartaj Virk ${ }^{\mathrm{b}}$, \\ Mahesh Bikkina $^{\mathrm{b}}$, Fayez Shamoon ${ }^{\mathrm{b}}$
}

\begin{abstract}
Background: The aim of the study was to compare the efficacy and safety of bivalirudin versus unfractionated heparin (UFH) in patients with acute myocardial infarction who undergo percutaneous coronary intervention (PCI). Earlier trials comparing bivalirudin and UFH during PCI demonstrated that bivalirudin caused less bleeding with more stent thrombosis. Since then, adjunct antiplatelet strategies have evolved. Improved upstream platelet inhibition with potent P2Y12 inhibitors decreased the need for routine glycoprotein IIb/IIIa inhibitor (GPI), resulting in similar outcomes among UFH and bivalirudin. Therefore, the role of bivalirudin in modern PCI practices is questionable.
\end{abstract}

Methods: We utilized Cochrane Review Manager (RevMan) 5.3 to perform a meta-analysis of seven randomized controlled trials (RCTs) with 22,844 patients to compare bivalirudin to UFH in patients with acute myocardial infarction requiring revascularization.

Results: There was no difference between bivalirudin and UFH regarding major adverse cardiac events (MACE), risk ratio (RR) 0.99, $95 \%$ confidence interval $(\mathrm{CI}) 0.87-1.12 ; \mathrm{P}=0.83)$ or cardiovascular mortality (RR 0.87, 95\% CI $0.71-1.07 ; \mathrm{P}=0.18$ ). Bivalirudin increased acute stent thrombosis (RR 2.77, 95\% CI 1.49 - 5.13; P = 0.001 ), which was only significant among ST-elevation myocardial infarction (STEMI) only trials. Bivalirudin caused less major bleeding (RR 0.66, 95\% CI $0.49-0.90 ; \mathrm{P}=0.007$ ), which was negated when GPI was used provisionally (RR $0.93,95 \%$ CI $0.64-1.33 ; \mathrm{P}=0.67$ ).

Conclusions: Among patients with acute myocardial infarction who underwent PCI, bivalirudin and UFH demonstrated similar MACE

Manuscript submitted July 30, 2019, accepted August 12, 2019

aDepartment of Cardiology, Cape Fear Valley Medical Center, Campbell University, Fayetteville, NC, USA

bSt Joseph's Health, New York Medical College, Paterson, NJ, USA

'Thomas Jefferson University Hospital, Philadelphia, PA, USA

dDepartment of Cardiology, Phoebe Putney Memorial Hospital, Albany, GA, USA

${ }^{e}$ Corresponding Author: Rana Garris, St Joseph's Health, New York Medical College, 703 Main St, Paterson, NJ 07503, USA.

Email: ray201603@gmail.com

doi: https://doi.org/10.14740/cr921 and cardiovascular mortality. Bivalirudin increased acute stent thrombosis, which was more remarkable among STEMI. Bivalirudin decreased major bleeding, but this benefit was negated when GPI was used provisionally.

Keywords: Bivalirudin; Heparin; STEMI; Percutaneous coronary angiography

\section{Introduction}

In patients with acute coronary syndrome, pharmacological modulation of both the coagulation cascade and platelet activation are essential components of percutaneous coronary angiography (PCI) [1]. Unfractionated heparin (UFH) is the mainstay anticoagulant in PCI, yet it can be unreliable. The efficacy of UFH is limited by its nonspecific binding, platelet activation, variable bioactivity, and its inability to inhibit clotbound thrombin [2]. Outside of its role in forming the fibrin clot, thrombin is a key activator of platelets, which release adenosine diphosphate (ADP) and thromboxane A2, mediators of platelet adhesion. P2Y12 binding of ADP facilitates the interaction of fibrinogen and glycoprotein IIb/IIIa, resulting in platelet aggregation and clot stabilization. Modulation of this pathway by glycoprotein IIb/IIIa inhibitors (GPI) facilitates coronary reperfusion, decreasing PCI-related reinfarction and death. Trials have demonstrated that although GPI improves the efficacy of UFH, this is at the cost of increased bleeding [3]. P2Y12 receptor antagonists are also important mediators in revascularization. Unequivocally, clopidogrel improves coronary artery patency and cardiovascular outcomes, yet its variable bioavailability and suboptimal early platelet inhibition are problematic $[4,5]$. Limitations of these PCI adjuncts led to the search for more predictable therapies [2]. In 1995, a landmark study guided the approval of bivalirudin as an alternate to UFH during PCI [6]. Bivalirudin is a 20 -amino-acid peptide that mimics hirudin, a naturally occurring direct thrombin inhibitor. It binds thrombin at two sites, which are interconnected by a cleavable polyglycine chain that allows simultaneous binding of fibrinogen. This unique mechanism is independent of antithrombin III, allowing for direct inhibition of both free and clot-bound thrombin. Bivalirudin demonstrates linear dosedependent activity, resulting in more predictable pharmokinet- 


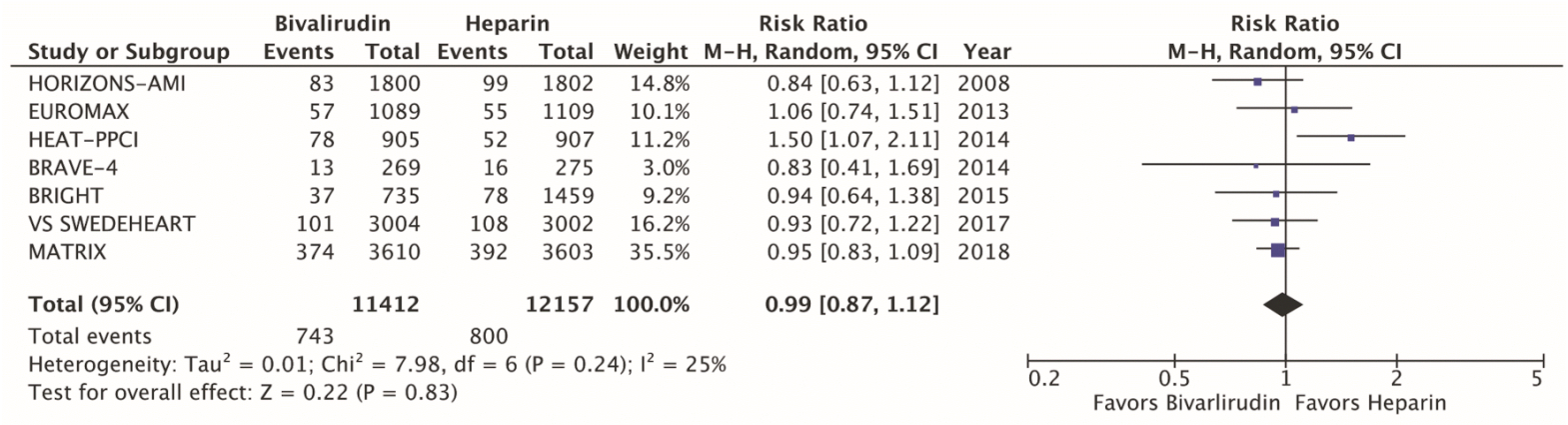

Figure 1. Forest plots of meta-analysis comparing MACE between bivalirudin and heparin arms. MACEs include composite death, myocardial infarction, and stroke. MACEs: major adverse cardiac events.

ics than UFH. Bivalirudin is also associated with less bleeding, attributed to its short half-life without the risk of platelet factor-4 antibody-induced thrombocytopenia [2]. Early trials demonstrated that bivalirudin was equally efficacious with less bleeding than UFH, despite its predilection for stent thrombosis $[7,8]$. While the economic benefit of bivalirudin exceeds that of UFH and routine GPI, bivalirudin costs hundreds more than UFH alone [9]. UFH monotherapy became more effective with the introduction of ticagrelor and prasugrel $[10,11]$. In comparison to clopidogrel, preloading with these P2Y12 inhibitors resulted in more potent platelet inhibition, which decreased the need for routine GPI. Consequently, provisional GPI use negated the bleeding benefit of bivalirudin [12]. Two recent trials performed by modern PCI practices with potent P2Y12 inhibitors and provisional GPI resulted in similar net adverse clinical events (NACE) among bivalirudin and UFH $[13,14]$. Yet, the observed overall reduction in stent thrombosis and bleeding may also be attributable to the inclusion of non-ST-elevation myocardial infarction (NSTEMI) and radial approach. The goal of this meta-analysis to assess the current role of bivalirudin in PCI.

\section{Materials and Methods}

We conducted a meta-analysis under the guidance of the Preferred Reporting Items for Systematic Reviews and MetaAnalyses (PRISMA) and the Cochrane Handbook of Systemic Reviews $[15,16]$. The present study complied with the Declaration of Helsinki and was approved by the institutional review board. Electronic databases were searched for the randomized controlled trials (RCTs) that assessed bivalirudin versus UFH in patients with acute myocardial infarction who underwent PCI. We used the following individual and/or combined search terms: "bivalirudin", "unfractionated heparin", "stent", "percutaneous coronary intervention", "acute coronary syndrome", and "ST-segment elevation myocardial infarction". The search was conducted from 2000 to 2018. We identified 674 records, of which 32 duplicates were removed and 642 records screened. Of these, 619 were excluded based on title and/or abstract and 23 full text articles were reviewed for eligibility. We excluded 16 records that were conducted in predominantly $(>50 \%)$ NSTEMI, unstable angina or elective PCI. Both
STEMI and NSTEMI trials were included among patients who presented within $24 \mathrm{~h}$ of symptom onset with electrocardiographic changes and elevated cardiac enzymes.

We utilized Cochrane Review Manager (RevMan) 5.3 to perform this meta-analysis with the Mantel-Haenszel method to analyze dichotomous data, measuring the risk ratio (RR) with a $95 \%$ confidence interval (CI). The Cochrane Q test was used to assess heterogeneity between the trials. Heterogeneity was quantified by I-squared. A random-effects model was used for all outcomes, and subgroup analyses were performed for identifiable variances of clinical significance. Funnel plots were created to assess for publication bias, and the Cochrane GRADE tool was used to estimate the level of outcome evidence. (Supplementary Index, www.cardiologyres.org).

The primary efficacy endpoint was the incidence of MACE, which include death, myocardial infarction, and stroke at 30 days (Fig. 1). Secondary efficacy endpoints were cardiovascular mortality at 30 days (Fig. 2) and stent thrombosis with subgroup analysis of acute $(<24 \mathrm{~h})$ versus subacute (30 days) and STEMI versus NSTEMI (Fig. 3). Secondary safety endpoints were major bleeding at 30 days with subgroup analysis of bleeding definitions and provisional versus routine use of GPI (Fig. 4 and Supplementary Index, www.cardiologyres.org).

\section{Results}

We identified a total of seven RCT that met the inclusion criteria, yielding a total of 22,844 patients. Table 1 highlights the trial characteristics and the Supplementary Index (www.cardiologyres.org) provides more description [7, 8, 12-14, 17-19]. The primary and secondary outcomes are delineated in Figures 1 - 4. There was no difference among bivalirudin and UFH in regards to MACE (RR $0.99,95 \%$ CI $0.87-1.12 ; \mathrm{P}=0.83$ ) or cardiovascular mortality (RR 0.87, 95\% CI 0.71 - 1.07; P = $0.18)$. There was an increased risk of acute stent thrombosis with the use of bivalirudin (RR 2.77, 95\% CI $1.49-5.13$; P $=0.001)$, while subacute $(\mathrm{RR} 1.15,95 \%$ CI $0.60-2.21 ; \mathrm{P}=$ 0.68 ) and overall (RR 1.49, 95\% CI $1.00-2.22 ; \mathrm{P}=0.05$ ) stent thrombosis were insignificant. In a subgroup analysis, STEMI trials appeared to have more significant stent thrombosis (RR $1.98,95 \%$ CI $1.06-3.69 ; \mathrm{P}=0.03$ ) than trials including NSTEMI (RR 1.13, 95\% CI $0.70-1.85 ; \mathrm{P}=0.62)$. Bivalirudin sig- 


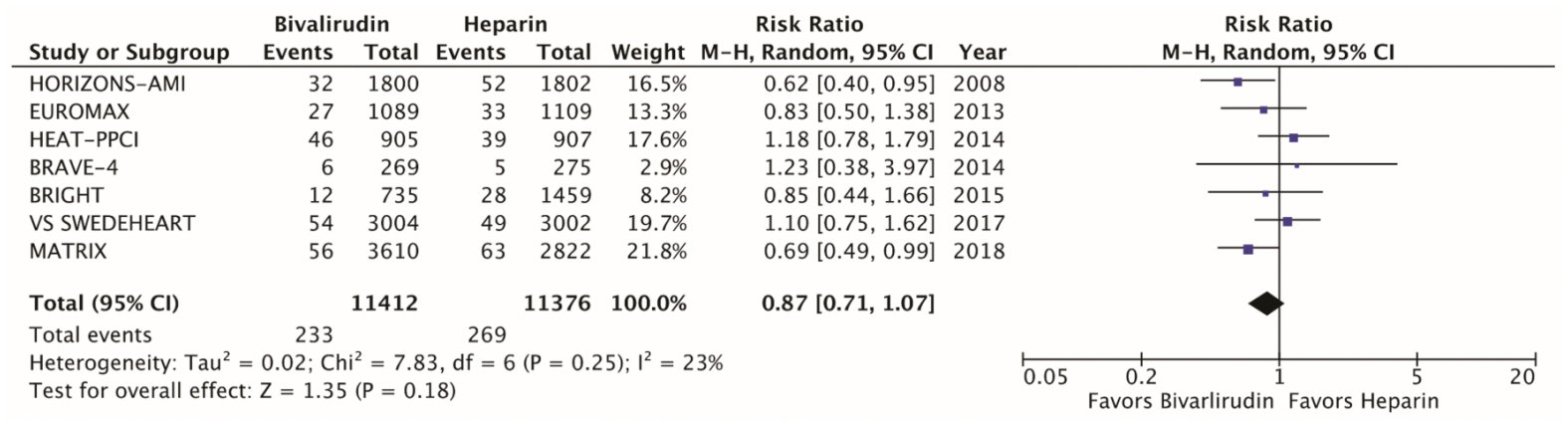

Figure 2. Forest plots of meta-analysis comparing cardiovascular mortality between bivalirudin and heparin. Cardiovascular mortality includes death due to acute myocardial infarction, pericardial tamponade, arrhythmia, peri-procedural complications or stroke.

nificantly reduced overall major bleeding as compared to UFH (RR 0.66, 95\% CI $0.49-0.90 ; \mathrm{P}=0.007)$. This was negated with provisional GPI use (RR 0.93, 95\% CI $0.64-1.33$; P < 0.67 ) in comparison to routine (RR $0.49,95 \%$ CI $0.37-0.64$; $\mathrm{P}<0.00001)$. Subanalysis suggested that major bleeds were more likely among trials that used the liberal HORIZONS bleeding definition $[7,8]$.

\section{Discussion}

This meta-analysis demonstrated that MACE and cardiovascular mortality were similar among bivalirudin and UFH, which can be explained by reinfarction and bleeding as drivers of these outcomes. HORIZONS-AMI, EUROMAX, and MATRIX showed that despite increased rates of bivalirudin stent thrombosis, bivalirudin decreased MACE and/or cardiovascular mortality [7, 19]. NACE was also decreased by bivalirudin in HORIZONS-AMI, EUROMAX, and BRIGHT [7, 18, 19]. These findings were attributable to less bleeding without an observed increase in reinfarction. Overall, only 1 trial with increased stent thrombosis displayed more reinfarction. [17]. Prior evidence established this importance, as reinfarction doubled the risk of mortality and post-PCI bleeding increased death by 5 times [20].

The low reinfarction rates observed during stent thrombosis were attributable to GPI, which was used as bailout during revascularization complicated by heavy clot burden or inadequate reflow $[7,8,17]$. Less bailout was required when there was better upstream platelet inhibition with potent P2Y12 antagonists [12, 13, 19]. The advantage of prasugrel and ticagrelor in PCI has been previously substantiated $[10,11]$. BRAVE-4 showed no difference in stent thrombosis among prasugrel/bivalirudin versus clopidogrel/heparin, and required GPI bailout in only 3\% of patients [12]. Ticagrelor was used in nearly all VALIDATE-SWEDEHEART patients, which demonstrated overall lower rates of stent thrombosis and reinfarction with less GPI bailout than all prior trials [13]. Moreover, its cardiovascular outcomes were independent of whether ticagrelor was given $>2$ hours or $<2$ hours before PCI [13]. While early optimal platelet inhibition is a clinical limitation of clopidogrel, ticagrelor achieves maximum platelet inhibition within $1-3$ hours [5]. Rates of ischemic events in BRAVE-4 and VALIDATE-SWEDEHEART were similar to those observed in BRIGHT with tirofiban/UFH use. Yet, while the GPI arm of BRIGHT demonstrated more bleeding, there was no significant difference among bleeding rates in BRAVE-4 and VALIDATE-SWEDEHEART $[12,13,18]$. This suggested that ticagrelor and prasugrel reduced stent thrombosis similar to GPI without the added risk of increased bleeding.

Extended infusion times of bivalirudin may have also mitigated stent thrombosis, as seen in EUROMAX, BRIGHT, and, VALIDATE-SWEDEHEART. Although all of BRIGHT patients were preloaded with clopidogrel, only $4 \%$ required bailout with similar rates of stent thrombosis and reinfarction among bivalirudin and UFH. Conversely, the MATRIX Treatment Duration study found no benefit to post-PCI bivalirudin infusion; however, this study was limited by the unspecified bivalirudin infusion regimen with variable dosing and infusion times at physician discretion [19]. A subsequent meta-analysis showed that extended bivalirudin infusion decreased cardiac events and mortality [21]. Therefore, it is reasonable to consider that the extended bivalirudin infusion regimens may have altered the rates stent thrombosis and reinfarction.

Clot burden has been considered a significant predictor of bivalirudin stent thrombosis [17]. The performed subgroup analysis demonstrated that stent thrombosis was significantly increased among STEMI trials $(2.2 \%)$, but not those which included NSTEMI (1.2\%). NSTEMI also had better post-PCI thrombolysis in myocardial infarction (TIMI) flow than STEMI and required less bailout GPI (Supplementary Index, www. cardiologyres.org). While more data is needed to confirm this finding, this likely had notable clinical implications.

Bivalirudin caused fewer major bleeds than UFH, which can be further analyzed in regards to bleed definition, access site, and GPI use. Earlier trials used HORIZONS, TIMI, and GUSTO bleeding definitions, while later trials used predominantly the BARC definition. The performed subgroup analysis suggested that major bleeds occurred more frequently in trials that used HORIZONS, a more liberal definition that includes access bleeds and need for blood transfusion (Supplementary Index, www.cardiologyres.org). There was no apparent difference among TIMI and GUSTO versus BARC $3 \mathrm{a}-\mathrm{c}$ and BARC 5 bleeds [22]. Radial access has been shown to reduce mortality through a reduction in bleeding $[23,24]$. The MATRIX trial randomly assigned over 8,000 patients to radial or femoral ac- 


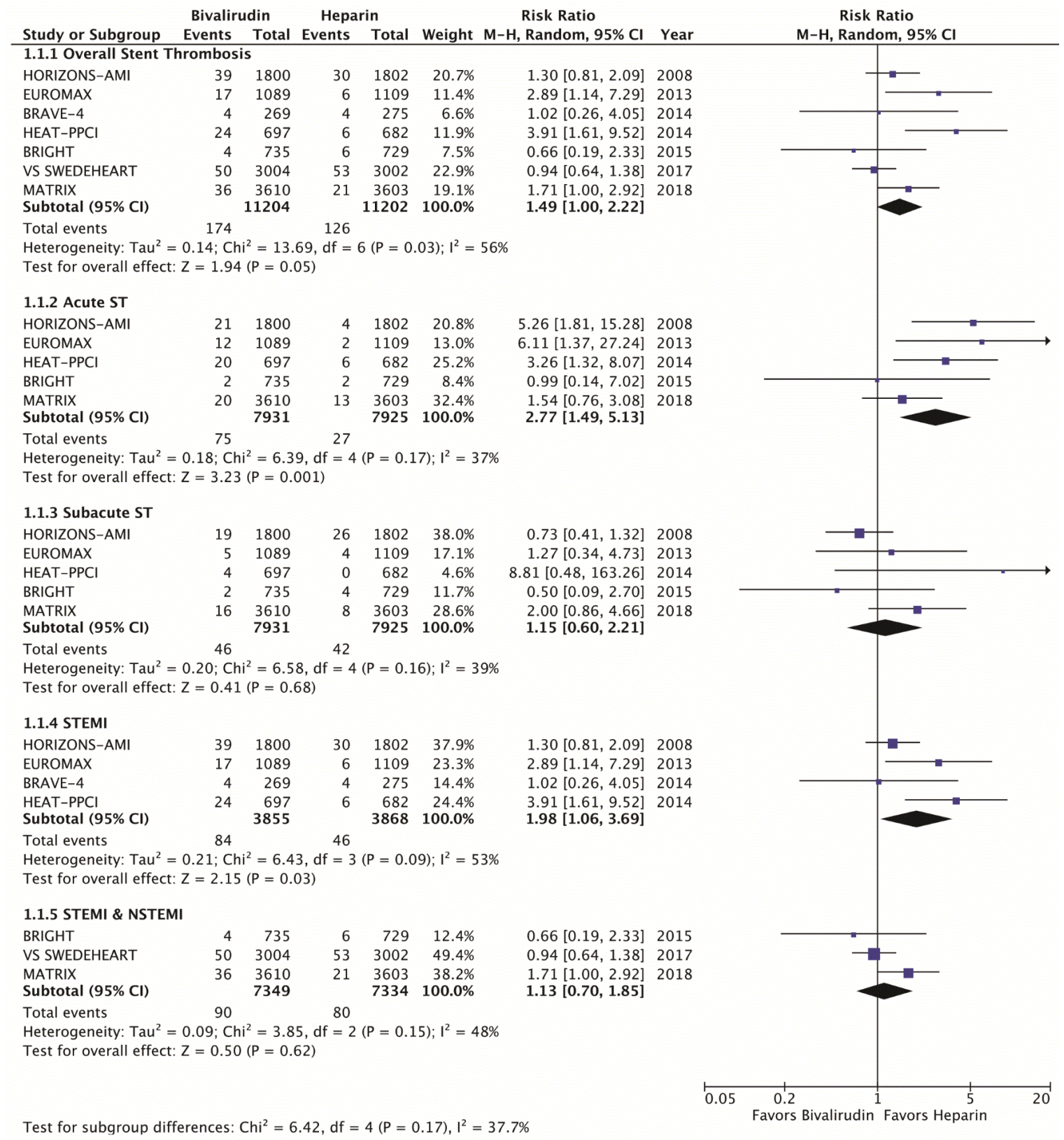

Figure 3. Forest plots of meta-analysis comparing stent thrombosis between bivalirudin and heparin. Acute stent thrombosis occurs within $24 \mathrm{~h}$ of PCl; subacute stent thrombosis occurs within 30 days of PCI. STEMI: ST-segment myocardial infarction; NSTEMI: non-ST segment myocardial infarction; PCl: percutaneous coronary intervention.

cess, and subsequently to bivalirudin or UFH, and showed that radial access reduced NACE and access site bleeds [14]. Trials BRIGHT, VALIDATE-SWEDEHEART, and HEAT-PPCI also predominantly used radial access $[13,17,18]$. While the bleeding benefit of bivalirudin was sustained in MATRIX and BRIGHT, it was negated in VALIDATE-SWEDEHEART and HEAT-PPCI.

GPI is potent antiplatelet therapy that improves ischemic outcomes, but it potentiates bleeding [3]. This analysis found that UFH and routine GPI resulted in significantly more major bleeds than bivalirudin $(4.7 \%$ versus $2.0 \%)$. When GPI was used provisionally, this difference was insignificant $(1.8 \%$ versus $2.1 \%$ ). Individually, early trials with routine or discretional GPI demonstrated that bivalirudin significantly reduced bleeds $[7,8]$. This was less apparent in subsequent trials where GPI was limited to bailout $[12,13,17]$. Prior studies have also demonstrated the GPI-dependent bleeding benefit of bivalirudin $[25,26]$. However, the pharmacokinetics of bivalirudin may confer additional benefit [2]. This was shown in BRIGHT, where bivalirudin decreased bleeding and NACE compared to both UFH/GPI and UFH monotherapy. Similar findings were demonstrated in MATRIX, where bivalirudin reduced bleeds, 
are at greater bleeding risk.

Patient comorbidities were also important considerations. Concurrent diseases such as anemia, kidney disease, diabetes, and hypertension predispose patients to bleeding, reinfarction, and death $[28,29]$. In EUROMAX, bivalirudin reduced bleeding by $3.4 \%$ when compared to UFH, yet more patients in the UFH group had anemia and chronic kidney disease (CKD). HORIZONS-AMI and EUROMAX had the highest number of patients with CKD, while subsequent trials often excluded these patients $[7,8]$. BRAVE-4 showed that adverse events occurred in $20.5 \%$ of patients with diabetes, versus $14.7 \%$ in patients without diabetes [12]. Patients with diabetes also had worse outcomes in HEAT-PPCI and EUROMAX [8, 17].

There were several limitations to this meta-analysis. Clinical and methodological differences common to systematic reviews resulted in unavoidable heterogeneity. Among these were the utilized drugs and administration protocols, procedural techniques, clot burden, facility expertise, and patient baseline characteristics. UFH dose varied from 70 to 100 units/ $\mathrm{kg}$ and some trials allowed for pre-procedural UFH bolus in both arms. The duration and dosing regimen of bivalirudin infusion also varied, as well as the type and timing of antiplatelet therapies. Access site and bleeding definitions were both confounders, the former of which was not stratified. Clot burden was another limitation. The majority of patients had STEMI, but some trials included NSTEMI. However, all patients had acute infarction requiring revascularization. Patient comorbidities, such as CKD, diabetes, hypertension, and prior myocardial infarction also contributed to patient variability. Lastly, trials varied in their sample sizes and locations. To address these limitations, we used a random-effects model, performed subgroup analysis, and evaluated the certainty of our evidence. [15].

\section{Conclusion}

In patients with acute coronary syndrome who underwent PCI, MACE and cardiovascular mortality were similar among bivalirudin and UFH. Bivalirudin increased the risk of acute stent thrombosis, specifically among STEMI patients. Less major bleeding was observed with bivalirudin, but this was negated with provisional GPI use.

\section{Acknowledgments}

None to declare.

\section{Financial Disclosure}

None to declare.

\section{Conflict of Interest}

None to declare.

\section{Informed Consent}

Not applicable.

\section{Author Contributions}

HP, RG, SB, PS, UR and RV contributed to study design, methods, data collection and analysis, writing; GM contributed to technical methods and editing; BAG contributed to technical methods, editing and mentorship; HV, MB and FS contributed to supervising attending physicians, mentorship and guidance.

\section{References}

1. Cimmino G, Fischetti S, Golino P. The two faces of thrombosis: coagulation cascade and platelet aggregation. Are platelets the main therapeutic target? J Thromb Circ Open Access. 2017;03(01):1-6.

2. Ramana RK, Lewis BE. Percutaneous coronary intervention in patients with acute coronary syndrome: focus on bivalirudin. Vasc Health Risk Manag. 2008;4(3):493-505.

3. Lincoff AM, Califf RM, Topol EJ. Platelet glycoprotein IIb/IIIa receptor blockade in coronary artery disease. J Am Coll Cardiol. 2000;35(5):1103-1115.

4. Vlaar PJ, Svilaas T, Damman K, de Smet BJ, Tijssen JG, Hillege HL, Zijlstra F. Impact of pretreatment with clopidogrel on initial patency and outcome in patients treated with primary percutaneous coronary intervention for STsegment elevation myocardial infarction: a systematic review. Circulation. 2008;118(18):1828-1836.

5. Damman P, Woudstra P, Kuijt WJ, de Winter RJ, James SK. P2Y12 platelet inhibition in clinical practice. J Thromb Thrombolysis. 2012;33(2):143-153.

6. Bittl JA, Strony J, Brinker JA, Ahmed WH, Meckel CR, Chaitman BR, Maraganore J, et al. Treatment with bivalirudin (Hirulog) as compared with heparin during coronary angioplasty for unstable or postinfarction angina. Hirulog Angioplasty Study Investigators. N Engl J Med. 1995;333(12):764-769.

7. Stone GW, Witzenbichler B, Guagliumi G, Peruga JZ, Brodie BR, Dudek D, Kornowski R, et al. Bivalirudin during primary PCI in acute myocardial infarction. N Engl J Med. 2008;358(21):2218-2230.

8. Steg PG, van 't Hof A, Hamm CW, Clemmensen P, Lapostolle F, Coste P, Ten Berg J, et al. Bivalirudin started during emergency transport for primary PCI. N Engl J Med. 2013;369(23):2207-2217.

9. Amin AP, Magnuson EA. Understanding the cost-effectiveness of bivalirudin. Heart. 2012;98(14):1037-1039.

10. Wiviott SD, Braunwald E, McCabe CH, Montalescot G, Ruzyllo W, Gottlieb S, Neumann FJ, et al. Prasugrel versus clopidogrel in patients with acute coronary syndromes. N Engl J Med. 2007;357(20):2001-2015.

11. Wallentin L, Becker RC, Budaj A, Cannon CP, Emanuelsson $\mathrm{H}$, Held C, Horrow J, et al. Ticagrelor versus clopidogrel in patients with acute coronary syndromes. 
N Engl J Med. 2009;361(11):1045-1057.

12. Schulz S, Richardt G, Laugwitz KL, Morath T, Neudecker J, Hoppmann P, Mehran R, et al. Prasugrel plus bivalirudin vs. clopidogrel plus heparin in patients with ST-segment elevation myocardial infarction. Eur Heart J. 2014;35(34):2285-2294.

13. Erlinge D, Omerovic E, Frobert O, Linder R, Danielewicz M, Hamid M, Swahn E, et al. Bivalirudin versus Heparin Monotherapy in Myocardial Infarction. N Engl J Med. 2017;377(12):1132-1142.

14. Valgimigli M, Frigoli E, Leonardi S, Vranckx P, Rothenbuhler M, Tebaldi M, Varbella F, et al. Radial versus femoral access and bivalirudin versus unfractionated heparin in invasively managed patients with acute coronary syndrome (MATRIX): final 1-year results of a multicentre, randomised controlled trial. Lancet. 2018;392(10150):835-848.

15. Higgins JPT, Green S. Cochrane Handbook for Systematic Reviews of Interventions. The Cochrane Collaboration. 2008.

16. Moher D, Liberati A, Tetzlaff J, Altman DG, the PRISMA Group. Preferred reporting items for systematic reviews and meta-analyses: the PRISMA statement. Ann Intern Med. 2009;151(4):264-269, W264.

17. Shahzad A, Kemp I, Mars C, Wilson K, Roome C, Cooper R, Andron M, et al. Unfractionated heparin versus bivalirudin in primary percutaneous coronary intervention (HEAT-PPCI): an open-label, single centre, randomised controlled trial. Lancet. 2014;384(9957):1849-1858.

18. Han Y, Guo J, Zheng Y, Zang H, Su X, Wang Y, Chen S, et al. Bivalirudin vs heparin with or without tirofiban during primary percutaneous coronary intervention in acute myocardial infarction: the BRIGHT randomized clinical trial. JAMA. 2015;313(13):1336-1346.

19. Valgimigli M, Frigoli E, Leonardi S, Rothenbuhler M, Gagnor A, Calabro P, Garducci S, et al. Bivalirudin or unfractionated heparin in acute coronary syndromes. N Engl J Med. 2015;373(11):997-1009.

20. Genereux P, Giustino G, Witzenbichler B, Weisz G, Stuckey TD, Rinaldi MJ, Neumann FJ, et al. Incidence, Predictors, and Impact of Post-Discharge Bleeding After Percutaneous Coronary Intervention. J Am Coll Cardiol. 2015;66(9):1036-1045.
21. Shah R, Matin K, Rogers KC, Rao SV. Effect of postprimary percutaneous coronary intervention bivalirudin infusion on net adverse clinical events and mortality: A comprehensive pairwise and network meta-analysis of randomized controlled trials. Catheter Cardiovasc Interv. 2017;90(2):196-204.

22. Mehran R, Rao SV, Bhatt DL, Gibson CM, Caixeta A, Eikelboom J, Kaul S, et al. Standardized bleeding definitions for cardiovascular clinical trials: a consensus report from the Bleeding Academic Research Consortium. Circulation. 2011;123(23):2736-2747.

23. Mehta SR, Jolly SS, Cairns J, Niemela K, Rao SV, Cheema AN, Steg PG, et al. Effects of radial versus femoral artery access in patients with acute coronary syndromes with or without ST-segment elevation. J Am Coll Cardiol. 2012;60(24):2490-2499.

24. Ferrante G, Rao SV, Juni P, Da Costa BR, Reimers B, Condorelli G, Anzuini A, et al. Radial versus femoral access for coronary interventions across the entire spectrum of patients with coronary artery disease: a metaanalysis of randomized trials. JACC Cardiovasc Interv. 2016;9(14):1419-1434.

25. Navarese EP, Schulze V, Andreotti F, Kowalewski M, Kolodziejczak M, Kandzari DE, Rassaf T, et al. Comprehensive meta-analysis of safety and efficacy of bivalirudin versus heparin with or without routine glycoprotein IIb/ IIIa inhibitors in patients with acute coronary syndrome. JACC Cardiovasc Interv. 2015;8(1 Pt B):201-213.

26. Cavender MA, Sabatine MS. Bivalirudin versus heparin in patients planned for percutaneous coronary intervention: a meta-analysis of randomised controlled trials. Lancet. 2014;384(9943):599-606.

27. Gargiulo G, Carrara G, Frigoli E, Vranckx P, Leonardi S, Ciociano N, Campo G, et al. Bivalirudin or heparin in patients undergoing invasive management of acute coronary syndromes. J Am Coll Cardiol. 2018;71(11):1231-1242.

28. Bhatti NK, Karimi Galougahi K, Paz Y, Nazif T, Moses JW, Leon MB, Stone GW, et al. Diagnosis and management of cardiovascular disease in advanced and end-stage renal disease. J Am Heart Assoc. 2016;5(8):e003648.

29. Singh M, Arora R, Kodumuri V, Khosla S, Jawad E. Coronary revascularization in diabetic patients: Current state of evidence. Exp Clin Cardiol. 2011;16(1):16-22. 